\title{
AVALIAÇÃO DA USABILIDADE DE SISTEMA NO MODO CLÁSSICO E DRAFITING E ANNOTATION DO AUTOCAD 2014
}

\author{
T. F. Costa ${ }^{1}$, D. F. Lima ${ }^{2}$, A. M. Sousa Junior ${ }^{3}$ \\ Universidade Federal do Semi-Árido \\ almir.mariano@ufersa.edu.br ${ }^{3}$
}

Submetido 04/03/2015 - Aceito 28/05/2017

DOI: $10.15628 /$ holos.2017.2825

\section{RESUMO}

A forma de projetar mudou significativamente desde que foi criado a ferramenta CAD em 1971, foi em 1982 que essa tecnologia começou a ser disseminada em larga escala. Esse acontecimento só foi possível devido o desenvolvimento de duas grandes ciências, a matemática e a computação. Com a popularização dessa ferramenta, muitas empresas surgiram ofertando software com a ferramenta CAD. A pioneira nesse setor é a empresa de software CAD AutoDESK, seu principal produto é o AutoCAD, sendo usado por milhares de arquitetos e engenheiros. A grande preocupação é no quesito segurança ao trabalhador, nesse setor, a facilidade de usar é a condicionante para gerar segurança aos usuários, isto é a definição de Usabilidade. Com relação a evolução do AutoCAD, diversas alterações foram feitas desde que foi criado, com a finalidade de contribuir para Usabilidade do sistema este software conta com dois modos de trabalho, o modo Classic e o DRAFITING \& ANNOTATION, sendo o segundo introduzido apenas em 2008, desde então perguntasse, até que ponto um modo supera o outro. Para avaliar foi utilizada uma metodologia baseado nos estudos de usabilidade e nas normas de Engenharia de Software, e percebesse que um modo não anula o outro, contudo, adiciona possibilidade.

PALAVRAS-CHAVE: Ferramenta CAD, Usabilidade, Drafiting \& Annotation, Clássico.

\section{SYSTEM USABILITY ASSESSMENT AND CLASSIC MODE AND AUTOCAD DRAFTING ANNOTATION 2014}

\begin{abstract}
The form of design has changed significantly since it was created the CAD tool in 1971 , was in 1982 that this technology began to be disseminated widely. This event was made possible by the development of two major science, mathematics and computing. With the popularity of this tool, many companies have emerged offering software with CAD tool. The pioneer in this sector is the software company CAD AutoDESK, its main product is AutoCAD, being used by thousands of architects and engineers. The major concern is in terms of security to workers in this sector, ease of use is the
\end{abstract}

condition to generate security to users, this is the definition of Usability . Regarding the evolution of AutoCAD, several changes have been made since it was created, in order to contribute to usability of the system this software has two working modes, Classic mode and the DRAFITING \& ANNOTATION, the second introduced only in 2008 since then asked to what extent a way overcomes the other. To evaluate one was based methodology used in usability studies and the rules of Software Engineering, and realized that a way does not cancel the other, only adds possibility.

KEYWORDS: CAD Tool, Usability, Drafiting \& Annotation, Classic. 


\section{FERRAMENTAS CAD}

A criação do computador impactou em todas as áreas de atuação humana, permitindo maior agilidade na execução das tarefas e até mesmo modificando a forma como são realizadas. Isso ocorreu devido o desenvolvimento de diversas ferramentas de trabalho que até então não existiam. Nas engenharias as ferramentas CAD (Computed-Aided Design ou Desenho Assistido por Computador), modificaram totalmente o modo como os projetos são concebidos. Diante de tanta opção que o mercado oferece perguntamos: Qual a melhor ferramenta? Qual a melhor forma de trabalhar?

Antes de prosseguir com a discussão, faremos um breve histórico sobre o surgimento das ferramentas CAD.

Sem os postulados da Matemática Euclidiana (criado por volta de 350 a.C), a criação das ferramentas CAD seria impossível. O primeiro software CAD foi criado na década de 60 do século XX, por Ivan Sutherland durante seu curso de pós-doutorado no MIT. O sistema foi chamado de "Sketchpad", no editor era possível criar e editar objetos em 2D, a grande novidade era a interação direta do usurário com o computador, através de uma espécie de caneta luminosa usada diretamente na tela para realizar o desenho.

Devido ao alto custo dos computadores, os softwares CAD eram usados apenas por empresas do setor aeroespacial e por grandes montadores de automóveis, como General Motors. Nessa época os softwares não eram comercializados livremente, sendo ainda objetos de pesquisa. Foi somente em 1970 que os softwares CAD passaram a ser comercializados livremente, nessa época já se havia desenvolvido o primeiro CAD 3D, sendo o pioneiro o Computer Aided Three Dimensional Interactive Application (CATIA) desenvolvido em 1977 pela empresa francesa Avions Marcel Dassault. A partir de 1980, com o primeiro PC (Personal Computer), desenvolvido pela IBM, a atenção dos desenvolvedores ficou voltada para essa classe de computadores.

Em 1982 foi criado o primeiro programa CAD para PC's, o AutoCad release 1, pela empresa Autodesk. Em 1985 é lançado uma segunda versão do CATIA. Na década de 90, em 1995 a empresa SolidWorks lança o SolidWorks 95 3D CAD, revolucionando o mercado de sistema computacional Windows NT. As ferramentas continuaram sendo melhoradas, e hoje diversas empresas ofertam tais software. Com a difusão da internet no final da década de 90 , os software CAD se tornam cada vez mais acessíveis, hoje os principais software CAD disponíveis e mais conhecidos no mercado são mostrados na tabela 1.

Tabela 1 - Principais software CAD.

\begin{tabular}{c|c}
\hline SOFTWARE & EMPRESAS \\
\hline SolidWORK & SolidWorks \\
\hline AutoCAD & AutoDESK \\
\hline CATIA & Dassault Systèmes \\
\hline REVIT & AutoDESK \\
\hline Pro ENGINEER & PTC \\
\hline AutoCAD MECHANICAL & AutoDESK \\
\hline CoreICAD & Corel \\
\hline Microstation & Bentley Systems
\end{tabular}


O que existe em comum entre todos esses softwares é que todos têm como base a ferramenta CAD tendo como distinção apenas a aplicação ao qual se destina. A escolha de uma das ferramentas citadas depende diretamente do tipo de aplicação e da área de atuação. $O$ SolidWorks, CATIA e ProEngineer são aplicações voltadas para engenheiros e designer de peças mecânicas com foco em desenho, modelagem e simulação, o AUTOCAD MECHANICAL incorpora funcionalidades do AutoCad além de bibliotecas de peças e ferramentas baseadas em padrões para ajudar a automatizar tarefas comuns de CAD mecânico e acelerar o processo de desenho mecânico. O AutoCad e o Revit parecem possuir as mesmas funcionalidades, ambos são utilizados na concepção de projetos de Engenharia e Arquitetura. Por ser o primeiro software CAD desenvolvido para computadores pessoais ( $P C^{\prime} s$ ), o AutoCad se consolidou como uma ferramenta prática de desenho e documentação de projetos. Entretanto, além das ferramentas CAD, um novo salto é dado no que diz respeito a forma de projetar e modelar objetos, surge então a ferramenta BIM (Modelagem de Informação de Construção) onde enquanto o projetista desenha as representações bidimensionais, a ferramenta modela uma representação tridimensional. O software AutoCAD REVIT foi desenvolvido tendo como base essa tecnologia, é um aplicativo individual que inclui recursos para projeto de arquitetura, de construção e de engenharia estrutural.

Desde que foi lançado em 1982 o AutoCAD sofreu diversas alterações, evoluindo até chegar ao modelo atual. A maior mudança ocorreu em 2008, quando a Autodesk incluiu no software uma nova forma de visualizar seus comandos e funções, o modo Drafting \& Annotation (Anotação e Elaboração). A partir de então as novas versões incluíam dois ambientes de desenho: - AutoCAD Classic e o Drafting \& Annotation. Essa modificação atingiu tanto o ambiente de desenho 2D quanto o 3D. No modo Classic os ícones eram dispostos em barras de tarefas, e nas laterais, as outras funcionalidades são encontradas nos menus: inserir; ferramentas; dimensão dentre outras. Com a atualização foi incorporado o conceito de guias, onde as ferramentas do software são agrupadas pelo critério de uso, nesse modo além dos ícones foi colocado o nome dos ícones onde é possível ver como utilizar cada ferramenta.

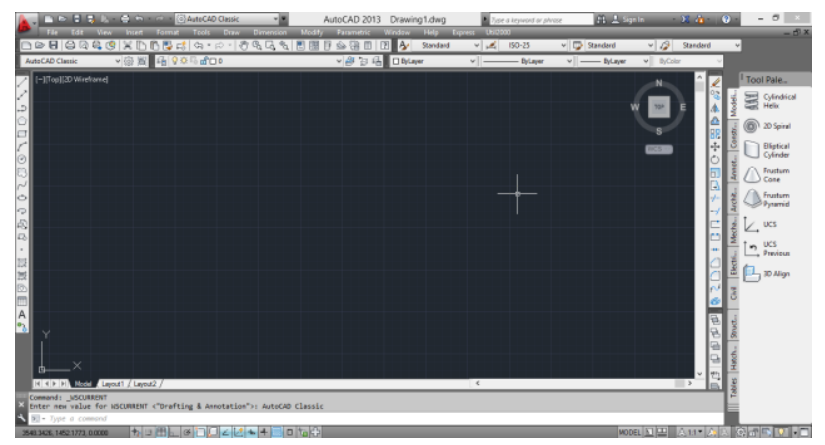

a) Interface do AutoCAD Classic

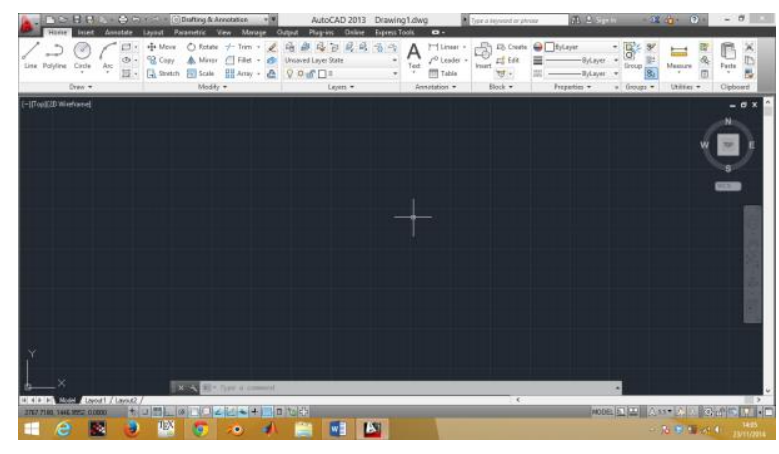

b) Interface do Drafting \& Annotation

No modo Clássico em geral os usuários trabalham com comandos no teclado enquanto que no ambiente de Desenho e Anotação o usuário opta por trabalhar com mais frequência utilizando o mouse, em ambos os ambiente os usuários tem o feedback de suas ações a partir da linha de comandos do software localizado na parte inferior da interface gráfica onde o software se comunica com o usuário informando as funcionalidades de cada função selecionada bem como possíveis erros. 
A partir de 2008 todas as atualizações mantem os dois modos para atender aos usuários antigos e os atuais. Essa versatilidade da Autodesk permitiu que o AutoCAD continuasse sendo a ferramenta mais usada nas Universidades, Institutos e Escritórios de Arquitetura e Engenharia. Diante disso até que ponto o ambiente Drafting \& Annotation supera o AutoCAD Classic?

Investigar esse problema, nos obriga a estudar o quão usual é cada ambiente. O que nos leva a um termo que vem sendo bastante discutido atualmente dentro da engenharia de sistema, Usabilidade de Sistema.

Segundo a American Journal of Industrial Medicine (outubro de 2002), um estudo feito por Tetsuya Nakazawa na Universidade de Chiba, no Japão, com mais de 25 mil trabalhadores de escritórios, revelou que quanto mais tempo um trabalhador passa diante de um computador, maior a probabilidade de desenvolver doenças físicas, transtornos mentais e distúrbios de sono. A UFRJ em seu editorial 164 feito em 19 de março de 2009 a partir de uma pesquisa encomendada pela intel sobre ergonomia, mostrou que dentre todos os riscos causados pelo mal uso do computador, tais como: queda de produtividade; problema de visão; lesão por esforços repetitivos; distúrbios osteomusculares relacionado ao trabalho devido a falhas no ambiente de trabalho, como: iluminação; ruído e temperatura, as interfaces dos programas são os fatores que mais afetam a saúde do trabalhador. "Além da questão física, a elaboração de programas sem uma interface que facilite ao usuário realizar suas tarefas contribui para o seu desgaste emocional e mental." (UFRJ editorial 164)

A Norma Regulamentadora que trata da adaptação do meio ambiente de trabalho as características psicofisiológicas dos trabalhadores de modo a promover o máximo de conforto, segurança e saúde, é a NR 17 - Ergonomia. Contudo esse instrumento legal não apresenta nenhuma exigência no que diz respeito à disposição das funções de um software dentro de sua interface. Entretanto com o avanço da Engenharia de Software as empresas de desenvolvimento tem se preocupado em fornecer tecnologias fáceis e intuitivas de operar, nasce a partir daí a Usabilidade de Sistema.

\section{USABILIDADE DE SISTEMAS}

Segundo Santos (2007) foi na Segunda Guerra Mundial que começou a haver uma preocupação com a interação homem máquina, quando a necessidade de agilidade e eficiência no manuseio de diversos equipamentos tais como: aviões; submarinos; radares; misseis, tornouse fator decisivo na guerra. Alcançava-se esses padrões construindo equipamentos coerentes com as características psicofisiologica dos operantes. Assim nascia a Ergonomia. Com o fim da guerra os estudos em ergonomia focou-se para os equipamentos do dia-a-dia. A partir da década de 70 a ergonomia passou a contribuir com sistemas interativos criando metodologias que identificassem problemas relativo ao contexto de uso de sistema. Esse conjunto de método ficou conhecido como Engenharia de Usabilidade ou simplesmente usabilidade.

Os principais estudiosos dessa área são: Miller; Bennet; Shackel ; Jakob Nielson; Bastien \& Scapin; Jordan; Shneiderman; Quensenbery e Cybis. Estudos envolvendo Engenharia de Usabilidade vem sendo feito desde 1970, dentre as obras mais citadas atualmente destacam-se as de Jakob Nielson, Claudia Dias e Walter de Abreu Cybis. Em uma busca feita no Google Acadêmico usando a palavra "usabilidade", as obras publicadas mais citadas entre 2003 e 2014 são:

1. Ergonomia e Usabilidade: conhecimento, método e aplicações. Walter de Abreu Cybis. Citado 286 vezes. 2007

2. Usabilidade na web: criando portais mais acessíveis. Cláudia Dias. Citado 285 vezes. 2003 
3. Engenharia de Usabilidade: uma abordagem mais econômica. Walter de Abreu Cybis. Citado 111 vezes. 2003

4. Usabilidade na web: projetando websites com qualidade. Jakob Nielson. Citado 127 vezes. 2007

De acordo com Shackel (1986), a definição de usabilidade foi introduzida por Miller em 1971 através de métricas relacionadas a "facilidade de uso". Miller identificou vários critérios para mensurar a facilidade de uso, como por exemplo: tempo de aprendizado, número de erros e tolerância à falhas Miller (1971).

Esse campo de estudo comporta diversas abordagens onde cada pesquisador desenvolve seus conceitos de usabilidade e estabelece métricas para avaliar o quão usual é o sistema. Na tentativa de reunir todas as ideias propostas por estes estudiosos, foram criadas algumas normas, que estabelece um padrão internacional para mensurar a usabilidade de um sistema. Dentre elas destacam-se a ISO 9241 REQUISITOS ERGÔNOMICOS PARA TRABALHO EM ESCRITÓRIO COM COMPUTADORES e a ISO 9126 ENGENHARIA DE SOFTWARE - QUALIDADE DE PRODUTOS.

A primeira é dividida em 17 partes, onde a parte 11 trata de Orientação sobre Usabilidade, a segunda é uma norma de Engenharia de Software que estabelece modelos de produção de softwares pensando na segurança humana, e traz um conjunto de métricas para avaliar a qualidade de Softwares, esta ISO é dividida em 4 partes onde a primeira traz os modelos de qualidade e as outras três as métricas de avaliação.

A ISO 9241-11 enfatiza que a usabilidade dos computadores é dependente do contexto de uso e que o nível de usabilidade alcançado dependerá das circunstâncias específicas nas quais o produto é usado. O contexto de uso consiste de usuários, tarefas, equipamentos (hardware, software e materiais), e do ambiente físico e social, pois todos esses podem influenciar a usabilidade de um produto dentro de um sistema de trabalho. Segundo ela, usabilidade é a medida na qual um produto pode ser usado por usuários específicos para alcançar objetivos específicos com eficácia, eficiência e satisfação em um contexto específico de uso.

Já a ISO 9126 estabelece um modelo de avaliação de qualidade de produtos de sistema a fim de produzir sistemas que alcancem a qualidade necessária para atingir as reais necessidades dos usuário. Nesse sentido analisa-se a qualidade interna, externa e de uso onde cada avaliação vai gerar feedback para os desenvolvedores e os desenvolvedores geram feedback para os usuários, contribuindo na especificação mais precisa dos requisitos. Cada requisito pode ser medido diretamente, indiretamente ou a partir de suas consequências, através das métricas estabelecidas. Esta norma categoriza os atributos de qualidade de software em 6 categorias: funcionalidade; confiabilidade; usabilidade; eficiência e manutenibilidade. Cada categoria possui um conjunto de subcategorias. Nesse trabalho estamos interessado apendas na usabilidade, que é definida pela ISO 9126 como a capacidade do produto de software de ser compreendido, aprendido, operado e atraente ao usuário, quando usado sob condições especificadas.

Dentro da categoria Usabilidade, definida pela ISO 9126, encontramos 5 subcategorias que também chamamos de métricas, são elas:

1. Inteligibilidade - Capacidade do produto de software de possibilitar ao usuário compreender se o software é apropriado e como ele pode ser usado para tarefas e condições de uso específicas.

2. Apreensibilidade - Capacidade do produto de software de possibilitar ao usuário aprender sua aplicação. 
3. Operacionalidade - Capacidade do produto de software de possibilitar ao usuário operálo e controlá-lo.

4. Atratividade - Capacidade do produto de software de ser atraente ao usuário.

5. Conformidade relacionada à usabilidade - Capacidade do produto de software de estar de acordo com normas, convenções, guias de estilo ou regulamentações relacionadas à usabilidade.

Além desses critérios estabelecidos pelas duas normas apresentadas, todos os estudiosos de usabilidade citados neste trabalho também desenvolveram individualmente um conjunto métricas que devem ser levado em consideração na avaliação de usabilidade de um sistema. Algumas delas são encontradas em todos os trabalhos, outras não.

\section{METODOLOGIA}

Com base nas divergências e convergências de opiniões dos estudiosos de usabilidade, Santos (2007) propõe um modelo de avaliação de usabilidade, baseado na ISO 9241 e ISO 9126. Este modelo está baseado em 6 critérios: facilidade de aprender; facilidade de relembrar; controle de erros; eficiência; eficácia e satisfação.

Conforme já foi citado, a usabilidade depende do contexto de uso, sendo que o contexto de uso é descrito pela descrição do usuários, tarefa, equipamento e ambiente. Fundamentado nesse argumento, Santos (2007) elaborou uma metodologia de avaliação de usabilidade que consiste em 4 etapas.

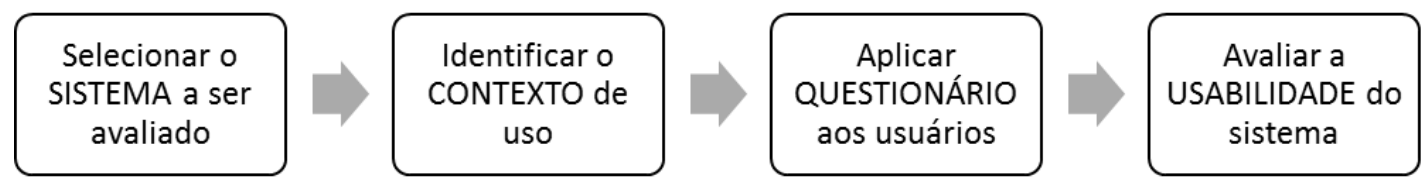

Entende-se por sistema, todo software que apresente iteração Homem x Maquina, essa metodologia aplica-se a software que já estão em funcionamento, não comtemplando softwares em desenvolvimento que em geral necessitam de modelos cíclicos.

O contexto é descrito pelos elementos especificados na ISO 9241: usuário; tarefa; equipamento e ambiente. $O$ questionário usado foi fundamentado nos 6 critérios propostos e encontra-se no Apêndice A, ele foi extraído da dissertação de mestrado de Santos (2007) autor da metodologia proposta e a avaliação será feita usando a lógica Fuzzi cujas operações matemáticas usadas também encontram descritas na referida dissertação.

Os 6 critérios propostos serão avaliados no questionário e estão distribuídos conforme mostra a tabela 2 .

Tabela 1 - Critérios propostos avaliados no questionário.

\begin{tabular}{l|c|c}
\hline \multicolumn{1}{c|}{ MÉTRICA } & NUMÉRO DE QUESTÕES & TOTAL DE QUESTÕES \\
\hline Facilidade de aprender & $1,2,3,4,7,8,9,10,11$ e 12 & 10 \\
\hline Facilidade de Relembrar & 13,14 e 15 & 03 \\
\hline Controle de erros & $16,17,18,19,20,21$, e 29 & 07 \\
\hline Eficiência & $22,23,24$ e 25 & 04 \\
\hline Eficácia & 26,27 e 28 & 03 \\
\hline Satisfação & 5,6 e 30 & 03 \\
\hline \multicolumn{2}{c|}{ Total }
\end{tabular}




\section{RESULTADOS E DISCUSSÕES}

A avaliação de usabilidade será aplicada no software AutoCAD com a finalidade de compreender qual ambiente é mais usual, o Drafting \& Annotation ou o Classic, do ponto de vista das normas e da metodologia usada. Para tanto, a identificação do contexto será feita de acordo com a ISO 9241, como segue abaixo.

\section{1 - Especificação dos usuários}

Os participantes da pesquisa são alunos do curso de Ciência e Tecnologia da Universidade Federal Rural do Semi-Árido - UFERSA campus Pau dos Ferros - RN, com no mínimo 1 ano e meio de graduação, idade média de 20 anos, experiência com uso de computadores de 8 anos e uso do sistema 1 ano. Conforme mostrou a pesquisa em geral os entrevistados usam o sistema eventualmente.

\section{2 - Especificação da tarefa}

$\mathrm{Na}$ perspectiva de promover um exercício para que a partir dele fosse preenchido o formulário de perguntas, foi desenvolvido um desenho de uma peça mecânica (Apêndice B) de modo que ele pudesse ser reproduzido, induzindo o uso das ferramentas básicas disponíveis no AutoCAD tais como: copiar, espelhar, mover, cortar, estender, cotas, leyers, escala, impressão, linhas, espessura, polígono e unidades. Os usuários do modo Clássico reproduzem o desenho usando a interface Clássica enquanto os usuário do modo Drafting, reproduzem usando a interface Drafting \& Anotation.

\section{3 - Especificação dos Equipamentos}

Os participantes da pesquisa usam o AutoCAD 2014, com o processo feito no laptop, em geral são processadores entre 2,5 e 3,0 GHz, 3 GB de RAM, 500 GB de HD e sistema operacional Windows 7, constituindo um ambiente homogêneo para todos os usuários.

\section{4 - Especificação do Ambiente}

O ambiente de estudo possuem iluminação e acústica adequada, o único inconveniente são as cadeiras que não são adaptativas gerando certo desconforto.

Foram selecionados 20 alunos, sendo $90 \%$ homens e $10 \%$ mulheres, que concluíram as disciplinas de CAD com média acima de 7,0. A pesquisa foi conduzida dentro do ambiente de estudo seguindo duas etapas: reprodução da desenho mecânico $2 \mathrm{D}$ e preenchimento do questionário. A quantidade de elementos da amostra é segundo a lógica Fuzzi suficiente para analisar comportamento, valores acima de 20 geram resultados constantes.

Os dados encontram-se tabulados no apêndice $\mathbf{C}$, onde foi calculada uma média para cada questão referente aos dois blocos, bloco 01 dados do modo Drafitting e bloco 02 dados do modo Classic. O resultado é mostrado no gráfico abaixo. 


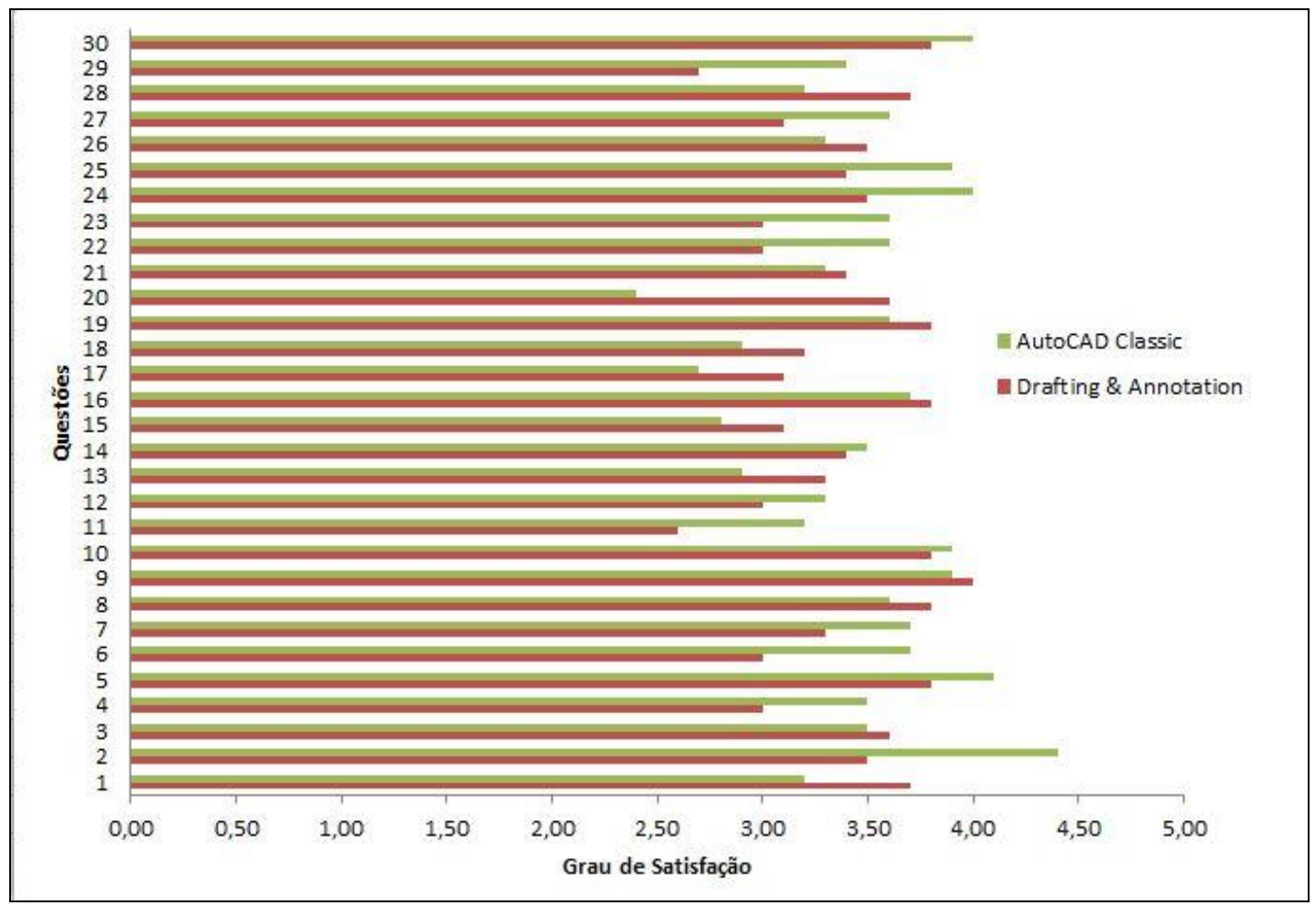

As métricas 1) facilidade de aprender; 2) facilidade de relembrar; e 3) eficácia, não fornecem resultados possíveis de fazer uma distinção entre quais modos é mais dominante, os resultados se mostraram parciais, gerando medidas positivas e negativas. Com relação as outras 3 métricas podemos observar os seguinte comportamentos:

1. Das 7 questões relativo a Controle de erros, 6 exibem resultados positivos no modo DRAFFING, mostrando que nos quesitos: retomada de erros; quantidade de erros; tempo de recuperação; satisfação em resolver os erros, esse gera mais eficiência, eficácia e satisfação aos usuários.

2. Contudo, no que diz respeito às métricas de eficiência e satisfação, os resultados foram imparciais ao modo Classic.

\section{CONCLUSÃO}

A evolução das ferramentas CAD permitiu grandes avanços no setor de projeto gerando precisão, aumentando a velocidade de elaboração e possibilitou a visualização de mais detalhes do projeto. Essa evolução só foi possível devido avanços na tecnologia da informação.

Desde que foi criado em 1971, e depois que foi lançado em larga escala em 1982, surgiram ao longo dos anos, até os dias atuais, diversos software portanto dessa ferramenta. Para se manter no mercado as empresas de desenvolvimento apostam cada vez mais na facilidade e intuição gerada pela interface, esse é um dos requisitos trabalhados dentro da engenharia de software. Com o AutoCAD não foi diferente, como o primeiro software CAD lançado em larga escala, ele ganhou popularidade e atualmente ocupa espaço na maioria dos laboratório de CAD das UNIVERSIDADES e INSTITUDOS, dentre as razões que levaram a isso está sua evolução.

Ao longo dos anos o AutoCAD sofreu grandes mudanças, como já foi citado, uma das maiores alteração foi a inserção do modo Draffiting em 2008. Como sabemos, as empresas estão 
cada vez mais apostando na ergonomia com a finalidade de gerar conforto e maior produtividade aos trabalhadores, isso não é diferente nos trabalhos de interação com softwares, nesse setor a usabilidade é um dos parâmetros a ser cumprido para gerar eficiência, eficácia e satisfação.

Pensando nisso este trabalho foi gerado como resultado de verificar até que ponto a alteração feita pela AutoDESK em 2008 contribui com a usabilidade do AutoCAD. Nessa avaliação fizemos uso de alguns instrumentos, tais como as normas ISO 9126 e ISO 9241, e os estudos de usabilidade mais importantes gerando um metodologia prática para ser aplicada em software em funcionamento.

O que afetou com a mudança, foi a forma como os erros são encarados pelos usuários, na escala de erros apresentados pelo sistema, recuperação de erros, tempo gasto para correção de erros, tempo gasto para refazer uma ação perdida por um erro, tratamento de erros e mensagem de erros, o modo Draffiting superou o modo Classic.

A disposição em forma de guias, possibilitou uma melhor visualização das ferramentas disponíveis no sistema, essa talvez seja a razão pela qual a nova forma de interação com o sistema contribui com o avanço da ferramenta. Entretendo percebemos que uma não supera a outra, somente soma possibilidades de interação, isso porque as vantagens não são tão divergentes.

Verifica-se que os dois ambientes de trabalhos não dispõem de insatisfação ou satisfação em nível máximo pelos entrevistados, variando de acordo com a percepção de cada participante, ou seja, enquanto um usuário prefere manusear o Drafiting e Annotation, outro opta pelo modo Clássico, cabendo a cada um utilizar aquele que julga fornecer melhores condições de usabilidade.

\section{REFERÊNCIAS}

Amaral, R.D.C., Filho, A.C.P. (2010). A evolução do CAD e sua aplicação em projetos de Engenharia. Simpósio de Mecânica Computacional, São João Del-Rei.

Bajerski, C. D., Schiffl, J. L., Silva, L. K., Sens, M. Y. O., Cordeiro, M. A., Eifler, R. A., Kubica S., Furusho, V. Y. (2009). Qualidade de software: Usabilidade. Companhia de Informática do Paraná. Recuperado em 15 de dezembro de 2015, de http://www.batebyte.pr.gov.br/modules/ conteudo/conteudo.php?conteudo $=1282$

Ferreira, S. B. L., Leite, J. C. S. do P. (2003). Avaliação da usabilidade em sistemas de informação: o caso do Sistema Submarino. Recuperado em 02 de janeiro de 2015, de http://www.scielo.br/scielo.php?script=sci_arttext\&pid=S14156555200

$3000200007 \&$ lng $=$ en\& $\mathrm{nrm}=$ iso

Infomações sobre REVIT. Recuperado em 15 de dezembro de 2014, de http://www.autodesk.com.br/products/revit-family/overview

Informações sobre AutoCAD Mechanical. Recuperado em 15 de dezembro de 2014, de http://www.autodesk.com/products/ autocad-mechanical/overview

Informações sobre AutoCAD. Recuperado em 15 de dezembro de 2014, de http://www.autodesk.com.br/products/autocad/overview

Informações sobre CATIA. Recuperado em 15 de dezembro de 2014, de http://www.3ds.com/products-services/catia/welcome/

Informações sobre ProEngineer. Recuperado em 15 de dezembro de 2014, de http://www.partnervision.com.br/softwares/ proengineer.html 
Informações sobre SolidWork. Recuperado em 15 de dezembro de 2014, de http://www.solidworksbrasil.com.br/sw/ newsdisplay.htm

Kreitzberg, C. B., Little A. (2015). A face Humana do software: Usabilidade na pratica. Recuperado em 27 de dezembro de 2015, de http://msdn.microsoft.com/pt$\mathrm{br} / \mathrm{magazine} / \mathrm{dd} 263095 . \mathrm{aspx}$

Patrias, K. (2007). Citing medicine: the NLM style guide for authors, editors, and publishers [Internet]. 2nd ed. Wendling DL, technical editor. Bethesda (MD): National Library of Medicine (US); - $\quad$ [atualizado em 15 set 2011; citado em 10 jan 2012]. Recuperado em 17 de julho de 2014, de http://www.nlm.nih.gov/citingmedicine

Prates, R. O., Barbosa, S. D. J. (2003). Avaliação de Interfaces de usuários: Conceitos e métodos. URISAN. Recuperado em 02 de dezembro de 2014, de http://www.urisan.tche.br/ paludo/material/IHM/Material/avaliacao.pdf

Prates, R. O., BARBOSA, S.D.J. (2010). Avaliação de interfaces de usuário - Conceitos e Método. Recuperado em 15 de maio de 2014, de http://www.urisan.tche.br/ paludo/material/IHM /Material/avaliacao.pdf

Santos, R. C. (2007). Desenvolvimento de uma metodologia para avaliação de Usabilidade de Sistemas utilizando a lógica Fuzzi baseado na ISO. Dissertação de mestrado, Faculdade de Economia e Finanças IBMEC, Rio de janeiro, RJ, Brasil.

Santos, R. L. G. (2006). Usabilidade de interfaces para sistemas de recuperação de informação na web. Tese de doutorado, PUC-Rio, Rio de Janeiro, RJ, Brasil. 


\section{APÊNDICE A}

\section{QUESTIONÁRIO PARA USABILIDADE DE SISTEMA}

AutoCAD 2014

Nome:

Sexo: ( ) Masculino ( ) Feminino

Idade:

Escolaridade:

Possui alguma limitação física? ___ (Sim/Não) Qual:

Tempo de experiência de uso de computador (em anos):

Tempo de experiência no uso do sistema (em anos):

Periodicidade do uso do sistema: ( ) Frequentemente ( ) Eventualmente ( ) Raramente

1. Ao completar uma tarefa com sucesso no sistema $p$ ela primeira vez, você achou :

( ) Muito Difícil ( ) Difícil ( ) Médio ( ) Fácil ( ) Muito Fácil

2. Qual foi sua primeira impressão ao utilizar o sistema:

( ) Muito Insatisfeito ( ) insatisfeito ( ) Médio ( ) satisfeito ( ) Muito satisfeito

3. O número de tentativas realizadas por você para aprender concluir uma tarefa, foi:

( ) Exagerada ( ) Muitas ( ) Médio ( ) Poucas ( ) Quase nenhuma

4. Com relação ao tempo para conseguir aprender a realizar uma tarefa com sucesso, você achou:

( ) Muito Demorado ( ) Demorado ( ) Médio ( ) Rápido ( ) Muito rápido

5. A interface do sistema lhe proporciona uma interação:

( ) Muito Insatisfatória () Insatisfatória ( ) Indiferente ( ) Satisfatória () Muito satisfatória

6. Ao realizar suas tarefas no sistema, com relação à clareza das mensagens, recuperação de erros, etc. Você se sente:

( ) Muito Desconfortável ( ) Desconfortável ( ) Médio ( ) confortável ( ) Muito Confortável

7. Com relação a facilidade de aprender uma tarefa, você se sente:

( ) Muito Insatisfeito ( ) Insatisfeito ( ) Médio ( ) Satisfeito ( ) Muito Satisfeito

8. Quantas são as maneiras diferentes que o sistema oferece para realizar a mesma tarefa, por exemplo: Caminho padrão vs

Teclas de atalhos, caminhos mais curtos, macros, botões específicos, etc. Você acha que:

( ) Existe um número muito baixo de possibilidades

( ) Existe um número baixo de possibilidades

() Existe um número razoável de possibilidades

( ) Existe um número alto de possibilidade

( ) Existe um número muito alto de possibilidade

9. Com relação a maneira mais rápida que você consegue realizar uma tarefa, comparando com a maneira padrão que o sistema oferece por padrão, você considera um ganho de produtividade:

( ) Muito Baixo ( ) Baixo ( ) Médio ( ) Alto ( ) Muito Alto

10. Com relação a flexibilidade que o sistema permite de executar suas tarefas de maneiras diferentes, como por exemplo: personalização de atalhos, valores, menus, macros, etc. Você se sente:

( ) Muito Insatisfeito ( ) Insatisfeito ( ) Indiferente ( ) Satisfeito ( ) Muito Satisfeito

11. O sistema é capaz de guia-lo através de sua execução com dicas, ajuda, avisos, etc.

( ) Nunca ( ) Raramente ( ) Algumas Vezes ( ) Na maioria das vezes ( ) Sempre

12. Ao completar uma tarefa com sucesso no sistema $p$ ela primeira vez, você achou:

( ) Muito Demorado ( ) Demorado ( ) Médio ( ) Rápido ( ) Muito Rápido

13. Após um período de tempo sem utilizar o sistema, você consegue relembrar como executar uma tarefa com:

( ) Muita Dificuldade ( ) Certa Dificuldade ( ) Um Esforço Médio ( ) Certa Facilidade ( ) Muita Facilidade

14. Toda vez que você precisa reutilizar o sistema com relação a relembrar sua utilização, você se sente:

( ) Muito Desconfortável ( )Desconfortável ( ) Médio ( ) Confortável ( ) Muito Confortável 1

15. Após um período de tempo sem utilizar o sistema, você consegue relembrar a utilização do sistema de forma:

() Muito Lenta ( ) Lenta ( ) Média () Rápido ( ) Muito Rápido

16. A quantidade de erros provocados $p$ elo sistema é:

( ) Muito Grande ( ) Grande ( ) Média ( ) Pequena ( ) Muito Pequena

17. Quando um erro o corre, a retomada ao funcionamento normal do sistema é:

( ) Muito Demorada ( ) Demorada ( ) Média ( ) Rápida ( ) Muito Rápida

18. Como você se sente com relação à quantidade de erros provocados pelo sistema:

() Muito Insatisfeito () Insatisfeito ( ) Indiferente () Satisfeito () Muito Satisfeito

19. A Quantidade de erros provocados $p$ elo sistema que causa alguma perda de informação ou retrabalho é:

( ) Muito Grande ( ) Grande ( ) Média ( ) Pequena ( ) Muito pequena

20. Quando um erro o corre, o tempo gasto para você retomar a execução da tarefa no sistema no ponto em que você parou é:

() Muito Demorado ( ) Demorado ( ) Média ( ) Rápido ( ) Muito Rápido

21. Como você se sente com relação à recuperação do erro por $p$ ar te do sistema, desfazer, refazer, voltar, salvar antes de fechar, 
etc.

() Muito Insatisfeito () Insatisfeito ( ) Indiferente () Satisfeito ( ) Muito Satisfeito

22. Como você considera a performance apresentada $p$ elo sistema:

( ) Muito Baixa ( ) Baixa ( ) Média ( ) Alta ( ) Muito Alta

23. Como você considera a velo cidade na realização das tarefas:

() Muito Demorado () Demorado ( ) Média ( ) Rápido ( ) Muito Rápido

24. Como você se sente com relação a produtividade do sistema:

( ) Muito Insatisfeito ( ) Insatisfeito ( ) Indiferente () Satisfeito () Muito Satisfeito

25. Como você se sente com relação à manter o sistema sob seu controle:

( ) Muito Insatisfeito ( ) Insatisfeito ( ) Indiferente ( ) Satisfeito ( ) Muito Satisfeito

26. Com relação a quantidade de passos par a realizar uma tarefa, você acha que é:

( ) Muito alto, consigo realizar a tarefa com eficácia muito baixa

( ) Alto, consigo realizar a tarefa com eficácia baixa

() Médio

() Baixa, consigo realizar a tarefa com eficácia alta

( ) Muito baixo, consigo realizar a tarefa com eficácia muito alta

27. Você consegue realizar uma tarefa qualquer no sistema de forma:

() Muito Demorado ( ) Demorado ( ) Média ( ) Rápido ( ) Muito Rápido

28. Como você se sente com relação à quantidade de passos para realizar uma tarefa no sistema:

() Muito Insatisfeito () Insatisfeito ( ) Indiferente ( ) Satisfeito ( ) Muito Satisfeito

29. As mensagens de erros apresentadas $p$ elo sistemas são:

( ) Muito Confusas ( ) Confusas ( ) Médias ( ) Esclarecedoras ( ) Muito Esclarecedoras

30. De uma forma geral, como se sente ao usar o sistema:

( ) Muito Insatisfeito ( ) Insatisfeito ( ) Indiferente () Satisfeito () Muito Satisfeito

\section{APÊNDICE B}

\section{Desenho proposto.}
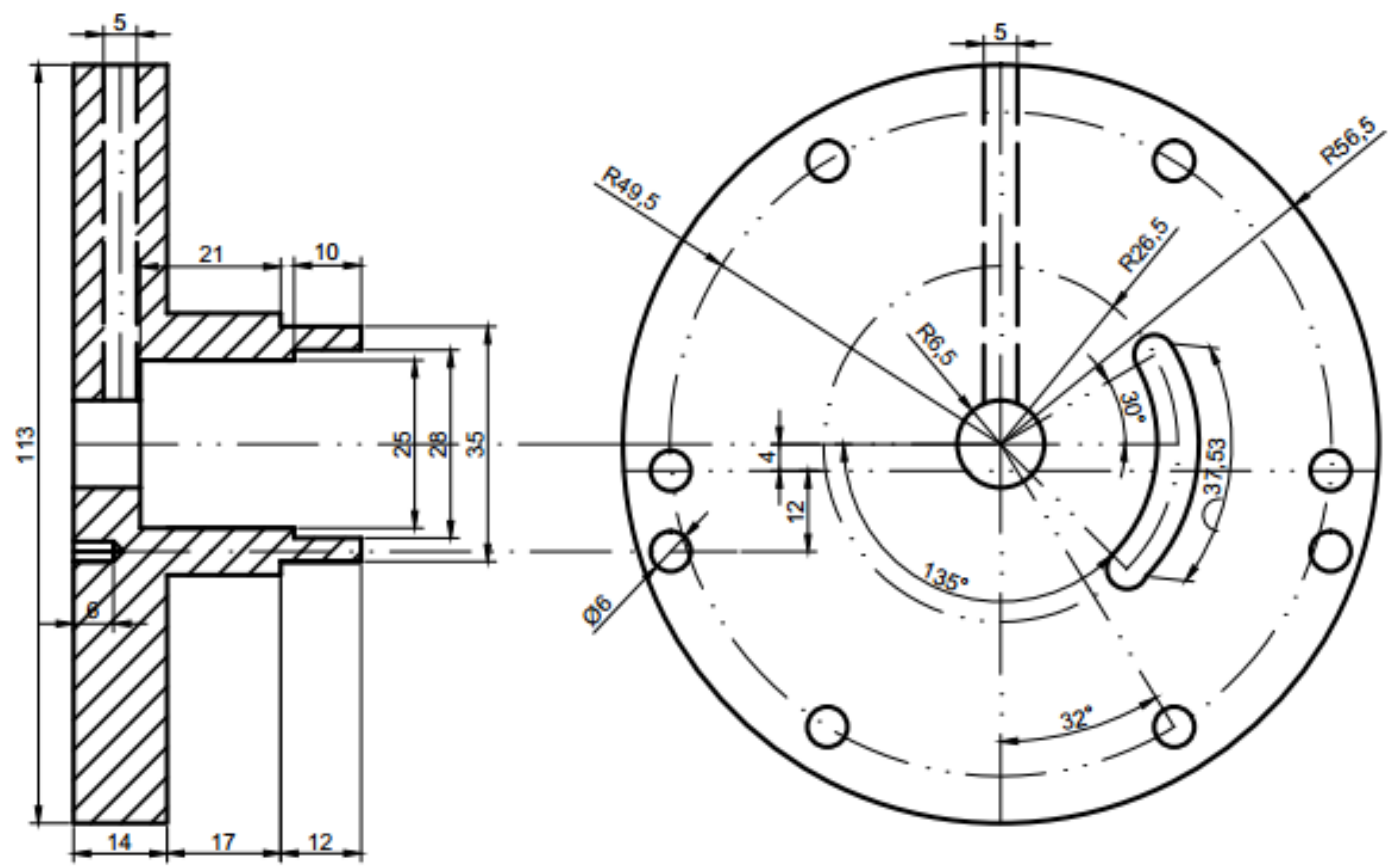


\section{APÊNDICE C}

Tabulação dos dados.

\begin{tabular}{|c|c|c|c|c|c|c|c|c|c|c|c|c|c|c|c|c|c|c|c|c|}
\hline & \multicolumn{10}{|c|}{ DRAFTING ANOTATION } & \multicolumn{10}{|c|}{ CLASSIC } \\
\hline $\begin{array}{c}\text { PERGUNTA } \\
\mathrm{S}\end{array}$ & 1 & 2 & 3 & 4 & 5 & 6 & 7 & 8 & 9 & 10 & 11 & 12 & 13 & 14 & 15 & 16 & 17 & 18 & 19 & 20 \\
\hline 1 & 4 & 3 & 3 & 4 & 4 & 4 & 4 & 3 & 4 & 4 & 3 & 4 & 3 & 4 & 2 & 3 & 4 & 3 & 4 & 2 \\
\hline 2 & 4 & 4 & 4 & 4 & 3 & 3 & 2 & 4 & 4 & 3 & 4 & 5 & 5 & 4 & 4 & 4 & 5 & 5 & 4 & 4 \\
\hline 3 & 4 & 2 & 3 & 4 & 4 & 4 & 4 & 3 & 4 & 4 & 4 & 4 & 3 & 4 & 3 & 3 & 4 & 3 & 4 & 3 \\
\hline 4 & 3 & 1 & 3 & 4 & 3 & 3 & 3 & 3 & 4 & 3 & 4 & 4 & 3 & 4 & 3 & 3 & 4 & 3 & 4 & 3 \\
\hline 5 & 4 & 4 & 4 & 4 & 3 & 4 & 4 & 4 & 4 & 3 & 4 & 4 & 4 & 4 & 4 & 5 & 4 & 4 & 4 & 4 \\
\hline 6 & 3 & 4 & 3 & 3 & 2 & 4 & 3 & 3 & 3 & 2 & 4 & 3 & 4 & 4 & 4 & 3 & 3 & 4 & 4 & 4 \\
\hline 7 & 3 & 1 & 3 & 4 & 3 & 5 & 4 & 3 & 4 & 3 & 4 & 4 & 4 & 4 & 3 & 3 & 4 & 4 & 4 & 3 \\
\hline 8 & 3 & 5 & 5 & 3 & 3 & 4 & 4 & 5 & 3 & 3 & 3 & 3 & 5 & 4 & 3 & 3 & 3 & 5 & 4 & 3 \\
\hline 9 & 5 & 4 & 3 & 4 & 4 & 5 & 4 & 3 & 4 & 4 & 3 & 3 & 4 & 4 & 5 & 4 & 3 & 4 & 4 & 5 \\
\hline 10 & 4 & 4 & 4 & 3 & 4 & 4 & 4 & 4 & 3 & 4 & 4 & 4 & 5 & 3 & 4 & 3 & 4 & 5 & 3 & 4 \\
\hline 11 & 2 & 2 & 3 & 2 & 3 & 3 & 3 & 3 & 2 & 3 & 3 & 3 & 3 & 3 & 4 & 3 & 3 & 3 & 3 & 4 \\
\hline 12 & 4 & 1 & 3 & 4 & 2 & 4 & 3 & 3 & 4 & 2 & 4 & 4 & 3 & 3 & 3 & 3 & 4 & 3 & 3 & 3 \\
\hline 13 & 3 & 3 & 3 & 3 & 4 & 3 & 4 & 3 & 3 & 4 & 3 & 3 & 2 & 3 & 3 & 4 & 3 & 2 & 3 & 3 \\
\hline 14 & 3 & 5 & 3 & 4 & 3 & 2 & 4 & 3 & 4 & 3 & 4 & 4 & 4 & 3 & 3 & 3 & 4 & 4 & 3 & 3 \\
\hline 15 & 3 & 5 & 3 & 3 & 2 & 3 & 4 & 3 & 3 & 2 & 3 & 2 & 3 & 3 & 3 & 3 & 2 & 3 & 3 & 3 \\
\hline 16 & 2 & 5 & 4 & 4 & 4 & 5 & 2 & 4 & 4 & 4 & 4 & 4 & 3 & 4 & 3 & 5 & 4 & 3 & 4 & 3 \\
\hline 17 & 1 & 2 & 3 & 4 & 4 & 4 & 2 & 3 & 4 & 4 & 3 & 3 & 2 & 2 & 3 & 4 & 3 & 2 & 2 & 3 \\
\hline 18 & 2 & 2 & 3 & 4 & 4 & 4 & 2 & 3 & 4 & 4 & 2 & 3 & 4 & 2 & 3 & 3 & 3 & 4 & 2 & 3 \\
\hline 19 & 4 & 4 & 3 & 4 & 4 & 5 & 3 & 3 & 4 & 4 & 5 & 4 & 4 & 2 & 4 & 3 & 4 & 4 & 2 & 4 \\
\hline 20 & 3 & 4 & 3 & 4 & 4 & 4 & 3 & 3 & 4 & 4 & 3 & 3 & 2 & 2 & 2 & 3 & 3 & 2 & 2 & 2 \\
\hline 21 & 4 & 4 & 2 & 4 & 4 & 3 & 3 & 2 & 4 & 4 & 4 & 3 & 2 & 4 & 4 & 3 & 3 & 2 & 4 & 4 \\
\hline 22 & 4 & 4 & 2 & 3 & 3 & 4 & 2 & 2 & 3 & 3 & 3 & 4 & 3 & 4 & 4 & 3 & 4 & 3 & 4 & 4 \\
\hline 23 & 3 & 4 & 2 & 4 & 2 & 4 & 3 & 2 & 4 & 2 & 4 & 4 & 3 & 3 & 4 & 4 & 4 & 3 & 3 & 4 \\
\hline 24 & 4 & 3 & 3 & 4 & 3 & 5 & 3 & 3 & 4 & 3 & 4 & 4 & 4 & 4 & 4 & 4 & 4 & 4 & 4 & 4 \\
\hline 25 & 4 & 4 & 2 & 4 & 3 & 4 & 4 & 2 & 4 & 3 & 4 & 4 & 4 & 4 & 4 & 3 & 4 & 4 & 4 & 4 \\
\hline 26 & 4 & 5 & 3 & 3 & 3 & 4 & 4 & 3 & 3 & 3 & 4 & 4 & 3 & 3 & 3 & 3 & 4 & 3 & 3 & 3 \\
\hline 27 & 3 & 3 & 3 & 3 & 3 & 4 & 3 & 3 & 3 & 3 & 4 & 4 & 3 & 3 & 4 & 4 & 4 & 3 & 3 & 4 \\
\hline 28 & 4 & 3 & 4 & 4 & 3 & 4 & 4 & 4 & 4 & 3 & 3 & 3 & 3 & 3 & 4 & 3 & 3 & 3 & 3 & 4 \\
\hline 29 & 4 & 1 & 3 & 3 & 3 & 2 & 2 & 3 & 3 & 3 & 3 & 3 & 4 & 4 & 3 & 3 & 3 & 4 & 4 & 3 \\
\hline 30 & 4 & 4 & 4 & 4 & 3 & 4 & 4 & 4 & 4 & 3 & 4 & 4 & 4 & 4 & 4 & 4 & 4 & 4 & 4 & 4 \\
\hline
\end{tabular}

